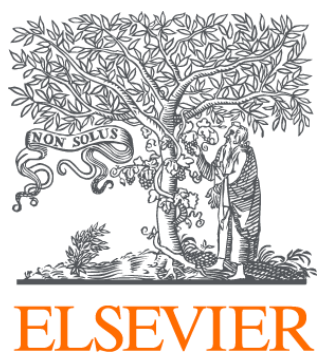

Since January 2020 Elsevier has created a COVID-19 resource centre with free information in English and Mandarin on the novel coronavirus COVID-

19. The COVID-19 resource centre is hosted on Elsevier Connect, the company's public news and information website.

Elsevier hereby grants permission to make all its COVID-19-related research that is available on the COVID-19 resource centre - including this research content - immediately available in PubMed Central and other publicly funded repositories, such as the WHO COVID database with rights for unrestricted research re-use and analyses in any form or by any means with acknowledgement of the original source. These permissions are granted for free by Elsevier for as long as the COVID-19 resource centre remains active. 


\section{OC.04 LIVER}

\section{C.04.1}

\section{HIGH LEVELS OF TRANSAMINASES AT ADMISSION PREDICT A SEVERE DISEASE COURSE IN COVID-19 HOSPITALIZED PATIENTS}

Pelizzaro F. ${ }^{* 1}$, Kitenge M.P., Maran F. ${ }^{1}$, Cocconcelli E. ${ }^{2}$, Balestro E. ${ }^{2}$, Spagnolo P. ${ }^{2}$, Cattelan A. ${ }^{3}$, Farinati F. ${ }^{1}$

${ }^{1}$ Department of Surgery, Oncology and Gastroenterology, Gastroenterology Unit, University of Padova, Padova, Italy, ${ }^{2}$ Department of Cardiac, Thoracic, Vascular Sciences and Public Health, Pneumology Unit, University of Padova, Padova, Italy, ${ }^{3}$ Infectious Disease Unit, University and Hospital of Padova, Padova, Italy

Background and aim: Abnormal liver function tests at hospital admission are frequently reported in patients with coronavirus disease 2019 (COVID-19), but their prognostic significance deserves further investigation. The aim of this study was to assess whether aspartate aminotransferase (AST) and alanine aminotransferase (ALT) abnormalities at admission predict disease course in patients hospitalized for COVID-19.

Materials and methods: In this single-center retrospective study, data of 188 patients admitted to the Infectious Disease Unit of the Padova University Hospital between February and May 2020 were collected. Patients were considered to have transaminases abnormalities when AST, ALT or both were above the upper limit of normal (ULN). The disease was considered as severe in case of intensive care unit (ICU) patient admission or death.

Results: At admission, 98 patients (52.1\%) had abnormal transaminases, but only 12 patients (6.4\%) showed levels above $3 \times$ ULN. Patients with severe disease showed higher AST (50 [IQR, 37-77] vs. 30 [IQR, 24-43] U/L, p<0.0001) and a trend to higher ALT (32 [IQR, 20-56] vs. 25 [IQR, 18-39] U/L, p=0.07) levels. Altered transaminases at baseline were associated with a higher probability of ICU transfer ( $40.8 \%$ vs. $15.6 \%, p=0.0002)$, mechanical ventilation ( $19.4 \%$ vs. $3.3 \%$, $\mathrm{p}=0.0005)$, non-invasive ventilation $(12.2 \%$ vs. $4.4 \%, \mathrm{p}=0.07)$, and longer hospital stay ( 9 vs. 7 days, $\mathrm{p}=0.002$ ). The risk of severe disease (transfer to ICU or death) was remarkably higher in patients with altered transaminases at admission (46.9\% vs. 15.6\%; p<0.0001) (Figure), and this was confirmed on multivariable logistic regression analysis after adjusting for sex, age, comorbidities, circulating inflammatory markers (neutrophil-to-lymphocyte ratio and C-reactive protein) and albumin levels with a five-fold higher risk (OR=5.17, 95\% CI 1.81-14.70; $\mathrm{p}=0.002)$.

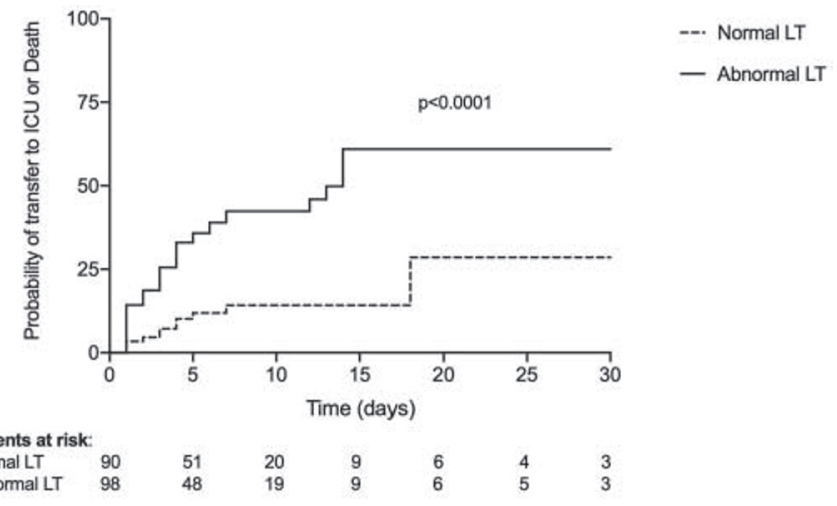

Conclusions: Hospitalized COVID-19 patients have frequently transaminases abnormalities at admission, and these alterations are able to independently predict a severe disease course.

\section{OC.04.2}

\section{PERSONALIZED DOSIMETRY STRONGLY AFFECT RESPONSE TO TARE AND SURVIVAL IN PATIENTS WITH INTERMEDIATE AND ADVANCED HCC}

Martelletti C. ${ }^{* 1}$, Ricotti A. ${ }^{2}$, Berchialla P. ${ }^{3}$, Marengo A. ${ }^{1}$, Morello E. ${ }^{1}$, Richetta E. ${ }^{4}$, Rocca R. ${ }^{1}$, Tabone M.

${ }^{1}$ Division of Gastroenterology, Mauriziano Hospital, Torino, Italy, ${ }^{2}$ Medical Direction of Hospital, Mauriziano Hospital, Torino, Italy, ${ }^{3}$ Department of Public Health and Pediatric, University of Torino, Torino, Italy, ${ }^{4}$ Department of Medical Physics, Mauriziano Hospital, Torino, Italy

Background and aim: There is a growing interest in the role of personalized dosimetry in the setting of Y90 transarterial radioembolization (TARE). The aim of this study was to analyze the effect of dosimetric variables of this technique on the outcome of patients with hepatocellular carcinoma (HCC).

Materials and methods: A total of 97 consecutive Child A5/6 cirrhotics patients with intermediate/advanced HCC treated with $90 \mathrm{Y}$ resin microspheres were retrospectively evaluated. Tumor response was assessed at 3 months by a quadriphasic TC and patients were divided into 4 groups according to mRECIST criteria. During the simulation phase the activities to be administered were calculated according to the MIRD multicompartimental formulas to determine the optimal tumoricide dose. The doses absorbed from the tumor were estimated by a PET-TC scan acquired 24 hours after the treatment. A p-value $<0.05$ was considered as significant.

Results: There were complete response (CR) in 25 patients, partial response (PR) in 30 patients, stable disease (SD) in 22 patients and progressive disease (PD) in 20 patients. Administered activities were: $1.8 \mathrm{GBq}(95 \% \mathrm{CI} 1.7-2), 1.8 \mathrm{GBq}$ (95\% CI 1.6-2), $1.7 \mathrm{GBq}(95 \% \mathrm{Cl}$ 1.5-1.9) and $1.65 \mathrm{GBq}(95 \% \mathrm{CI} 1.3-1.9)$ in CR, PR, SD and PD patients respectively ( $\mathrm{p}=0.358)$. Absorbed tumor doses were: $238 \mathrm{~Gy}(\mathrm{QR}$ 193-393), 190 Gy (IQR 153-269), 121 Gy (IQR 93-173) and 107 Gy (IQR 85-124) in CR, PR, SD and PD patients respectively $(\mathrm{p}<0.001)$. There were no significant differences in variables associated with
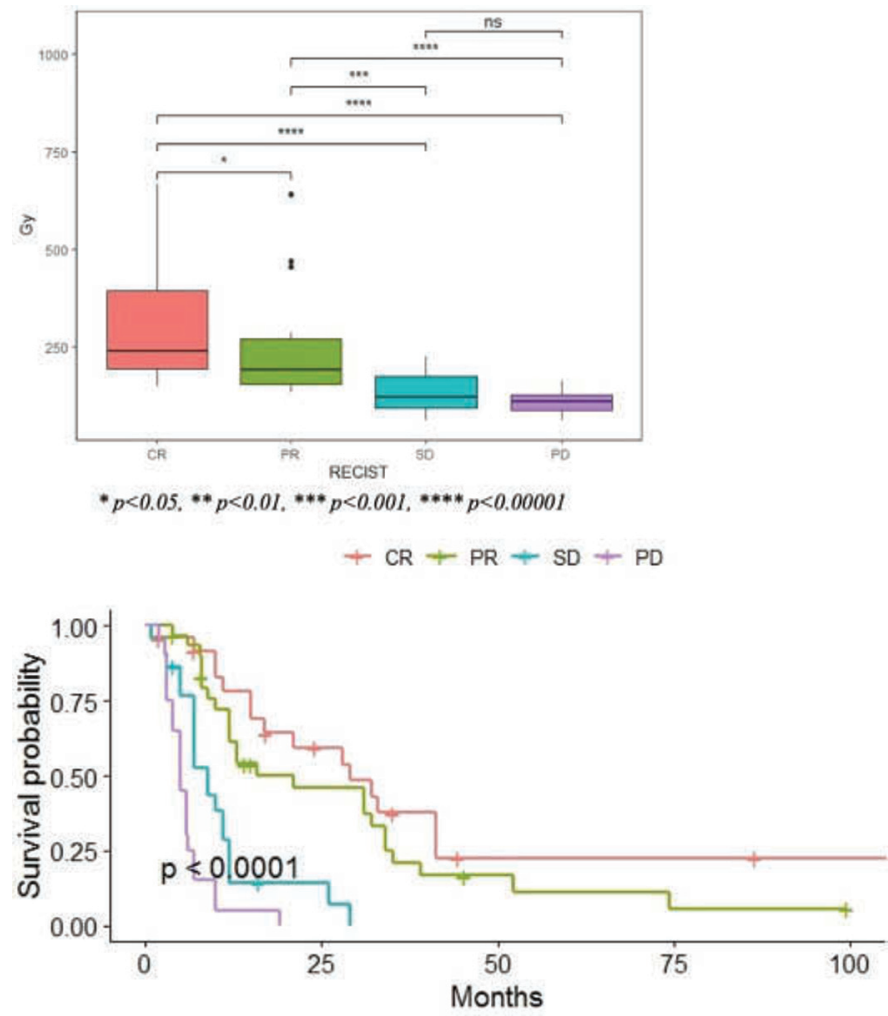